file: vectorfinal.tex

PACS numbers: $04.50 . \mathrm{Cd}$

\title{
Kaluza-Klein towers for real vector fields in flat space
}

\author{
Fernand Grard ${ }^{1}$, Jean Nuyts ${ }^{2}$
}

\begin{abstract}
We consider a free real vector field propagating in a five dimensional flat space with its fifth dimension compactified either on a strip or on a circle and perform a Kalaza Klein reduction which breaks $\mathrm{SO}(4,1)$ invariance while preserving $\mathrm{SO}(3,1)$ invariance. Taking into account the Lorenz gauge condition, we obtain from the most general hermiticity conditions for the relevant operators all the allowed boundary conditions which have to be imposed on the fields in the extra-dimension. The physical Kaluza-Klein mass towers, which result in a four-dimensional brane, are determined in the different distinct allowed cases. They depend on the bulk mass, on the parameters of the boundary conditions and on the extra parameter present in the Lagrangian. In general, they involve vector states together with accompanying scalar states.

1 fernand.grard@umons.ac.be, Physique Générale et Physique des Particules Elémentaires, Université de Mons - UMONS, 20 Place du Parc, 7000 Mons, Belgium

2 jean.nuyts@umons.ac.be, Physique Théorique et Mathématique, Université de Mons - UMONS, 20 Place du Parc, 7000 Mons, Belgium
\end{abstract}




\section{Introduction}

In previous publications [1, we have applied the Kaluza-Klein reduction procedure [2] to various fields propagating in a five dimensional space with the fifth dimension compactified either on a strip or on a circle.

Our approach is based on an exhaustive study of the hermiticity properties of the operators defining the masses. This results in the determination of all the sets of allowed field boundary conditions which have to be applied in the extra dimension. We have successively considered scalar and spinor fields in flat and warped spaces and have established in each case all the corresponding Kaluza-Klein towers. Our main result is that, apart from the usual towers where the masses (or masses squared) are regularly spaced (essentially linearly), there appears new types of towers with masses which are solutions of specific transcendental functions.

We consider here the case of a free real vector field in the five dimensional flat space and discuss its most general free Lagrangian and related equations of motions (see Sect.(2.1)). Requiring the hermiticity of the relevant operators, the basic boundary relations for the field are established in Sect.(2.2). Adopting a K-K reduction which breaks $\mathrm{SO}(4,1)$ covariance while preserving $\mathrm{SO}(3,1)$ covariance, the boundary relations for the reduced fields in the fifth dimension are derived (see Sect.(2.3)), from which, taking into account the restrictions imposed by the 5-dimensional Lorenz gauge condition, all the sets of allowed boundary conditions are obtained(see (2.4)). Some physical considerations about the locality of the boundary conditions and about the possible extension to a closed circle in the extra-dimension are given in Sect.(3.1) while the physical relevance of the mass scales which have to be adopted in a flat space is discussed in Sect.(3.2). A complete analysis of the boundary conditions leads to the appearance of both 4-dimensional vector and scalar states in the resulting Kaluza-Klein mass towers as seen in the 4-dimensional branes(see Sect.(41)).

Some physical consequences are discussed and the main results are summarized in the conclusions (Sect.(5)) 


\section{Real vector field in five dimensional flat space. Equations of motion. Kaluza-Klein reduction. Boundary relations and bound- ary conditions}

\subsection{Lagrangian. Equations of motion}

Let $B^{A}$ be a free real vector field propagating in a five-dimensional flat space

$$
x^{A}=\left\{\bar{x}, x^{5}\right\}=\left\{x^{\mu}, x^{5}\right\}, \mu=0,1,2,3, s=x^{5}
$$

with an infinite four dimensional Minkowski space $\bar{x}$ and the fifth dimension $s$ compactified on a strip $[0,2 \pi R]$ or on a circle of radius $R$. The metric is $\eta^{\mu \nu}=\operatorname{diag}(1,-1,-1,-1), \eta^{\mu 5}=0, \eta^{55}=-1$. The most general Lorentz invariant action, quadratic in the vector field $B^{A}$, contains, apart from the bulk mass $M$, two (real) free parameters $\lambda_{1}$ and $\lambda_{2}$

$$
\mathcal{A}=\int\left(\frac{1}{4} F_{A B} F^{A B}+\frac{\lambda_{1}}{2}\left(\partial_{A} B^{A}\right)^{2}+\frac{\lambda_{2}}{2}\left(\partial_{A} B_{B}\right)\left(\partial^{B} B^{A}\right)-\frac{M^{2}}{2} B_{A} B^{A}\right) d^{5} x
$$

with

$$
F_{A B}=\partial_{A} B_{B}-\partial_{B} B_{A} .
$$

Since the two factors of $\lambda_{1}$ and $\lambda_{2}$ are equal up to a divergence, the equations of motion (14) depend only on $\lambda$ with

$$
\lambda=\lambda_{1}+\lambda_{2}
$$

The so-called usual case corresponds to the choice $\lambda=0$ and we will focuss our attention on it. We will also discuss what we call the generalized case with $\lambda \neq 0$. Compared to the usual case, the generalized case involves, as we will explain later, essentially one more degree of freedom which is of scalar type. We will see that the five dimensional vector field leads, upon Kaluza-Klein reduction, to vector and scalar particles, mimicking, in some cases, 4-dimensional situations corresponding to a generalized case in the brane. 


\subsubsection{Equations of motion. Usual case $(\lambda=0)$}

The usual Lorentz invariant action (see however the discussion of the "Generalized case" below) corresponds to the particular choice $\lambda=0$

$$
\mathcal{A}=\int\left(\frac{1}{4} F_{A B} F^{A B}-\frac{M^{2}}{2} B_{A} B^{A}\right) d^{5} x
$$

and leads to the equations of motion

$$
\left(\square_{5}+M^{2}\right) B^{A}-\partial^{A}\left(\partial_{C} B^{C}\right)=0 .
$$

Usual Case $(\lambda=0)$ with $M^{2} \neq 0$

For $M^{2} \neq 0$, one deduces from the divergence of (6) the usual Lorenz condition

$$
\partial_{A} B^{A}=0
$$

and the equations of motion

$$
\left(\square_{5}+M^{2}\right) B^{A}=0
$$

which justify the interpretation of $M^{2}$ as the bulk mass squared of the transverse (Eq.(77)) massive (Eq.(8) ) vector field with four degrees of freedom.

Usual Case $(\lambda=0)$ with $M^{2}=0$

For $M^{2}=0$, the Lagrangian is invariant under the gauge transformation

$$
B^{A} \longrightarrow B^{A}+\partial^{A} \Lambda
$$

for any arbitrary $\Lambda$.

The Lorenz condition (77) can be obtained by a suitable gauge transformation

$$
\left(B^{\prime}\right)^{A}=B^{A}+\partial^{A} \Lambda
$$

leading to

$$
\begin{aligned}
& \square_{5} B^{\prime A}=0 \\
& \partial_{A} B^{\prime A}=0
\end{aligned}
$$


provided $\Lambda$ is chosen as

$$
\square_{5} \Lambda=-\partial_{A} B^{A}
$$

which defines $\Lambda$ up to an arbitrary function of null Dalembertian. In the five dimensional space, $B^{\prime}{ }^{A}$ has three transverse degrees of freedom. Indeed, the longitudinal degree of freedom can be eliminated by a further suitable gauge transformation (10) of null Dalembertian.

In the main part of the text, we will use, for the usual case $\lambda=0$, the equations (8) and (7) for all $M^{2}$.

\subsubsection{Equations of motion. Generalized case $(\lambda \neq 0)$}

The equations of motion in the generalized case (2)-(4), corresponding to $\lambda \neq 0$, are

$$
\left(\square_{5}+M^{2}\right) B^{A}+(\lambda-1) \partial^{A}\left(\partial_{B} B^{B}\right)=0 .
$$

Generalized Case $(\lambda \neq 0)$ with $M^{2} \neq 0$

For $\lambda \neq 0$ and $M^{2} \neq 0$, defining the new vector field $B^{\prime A}$ and the new scalar field $B^{\prime}$ by

$$
\begin{aligned}
\left(B^{\prime}\right)^{A} & =B^{A}+\frac{\lambda}{M^{2}} \partial^{A}\left(\partial_{L} B^{L}\right) \\
B^{\prime} & =\partial_{A} B^{A},
\end{aligned}
$$

one easily sees that they are the physical fields as they satisfy, from (14), the usual physical equations

$$
\begin{aligned}
\left(\square_{5}+M^{2}\right) B^{\prime A} & =0 \\
\partial_{A} B^{\prime A} & =0 \\
\left(\square_{5}+\frac{M^{2}}{\lambda}\right) B^{\prime} & =0 .
\end{aligned}
$$

Let us stress that the bulk mass squared of the vector field is $M^{2}$ while the bulk mass squared of the scalar field is multiplied by $1 / \lambda$.

Inversely, if $B^{\prime A}$ and $B^{\prime}$ are given such that they satisfy (16), then $B^{A}$ defined by

$$
B^{A}=B^{\prime A}-\frac{\lambda}{M^{2}} \partial^{A} B^{\prime}
$$


satisfies the initial equation (14). This shows that the physical fields $B^{\prime} A, B^{\prime}$ and the initial fields $B^{A}$ are equivalent ways of describing the theory.

Generalized Case $(\lambda \neq 0)$ with $M^{2}=0$

For $M^{2}=0$, the equations of motion in the generalized case $(\lambda \neq 0)$ reduce to

$$
\square_{5} B^{A}+(\lambda-1) \partial^{A}\left(\partial_{B} B^{B}\right)=0 .
$$

Let us note that the full gauge invariance (Eq.(9) with $\Lambda$ arbitrary) of the usual Lagrangian $(\lambda=0)$ is lost, but there is a remaining "partial gauge" invariance provided that

$$
\square_{5} \Lambda=0 .
$$

It is convenient to define the new fields $B^{\prime} A$ and $B^{\prime}$ by

$$
\begin{aligned}
\left(B^{\prime}\right)^{A} & =B^{A}+\partial^{A} \Sigma \\
B^{\prime} & =\partial_{A} B^{A}
\end{aligned}
$$

with $\Sigma$ chosen to satisfy

$$
\square_{5} \Sigma=(\lambda-1) \partial_{A} B^{A}
$$

Remark that $\Sigma$ is determined up to a function of null Dalembertian i.e. $\left(B^{\prime}\right)^{A}$ can still be changed by a partial gauge transformation.

The new fields then obey the equations

$$
\begin{aligned}
\square_{5} B^{\prime A} & =0 \\
\square_{5} B^{\prime} & =0 \\
\partial_{A} B^{\prime A} & =\lambda B^{\prime} .
\end{aligned}
$$

The interpretation of $B^{\prime}$ as a massless physical scalar field poses no problem. The interpretation of $B^{\prime A}$ as a massless vector field is more delicate as the Lorenz gauge condition is missing. In momentum space (with $p^{2}=0$ by (23) ), the five degrees of freedom of $B^{\prime}{ }^{A}$ decompose as follows. The divergence part $\partial_{A} B^{\prime} A$ is equivalent to the scalar $B^{\prime}$ by (25). Any longitudinal part (in the direction of $p^{A}$ ) is physically irrelevant as it can be removed by a suitable partial gauge transformation (19). The truly transverse part with three degrees of freedom describes a physical massless vector field. The transverse part is defined up to an arbitrary longitudinal part i.e. belongs to 
the equivalence class, in the mathematical sense, of fields differing by partial gauge transformations.

In the main part of the paper, a complete discussion of the usual case will be given. The changes which follow from the generalized case $(\lambda \neq 0)$ are outlined.

\subsection{Basic boundary relations}

\subsubsection{Basic boundary relations. Usual case $(\lambda=0)$}

In order to guarantee the reality of the bulk mass squared $M^{2}$ (see (8) and (11)), following the approach discussed and justified at great length in our preceding articles, [1], we impose that the operator $\square_{5}$ is an observable and hence hermitian (more precisely symmetric)

$$
\begin{aligned}
& (C, \mathrm{O} B)=(\mathrm{O} C, B) \\
& \mathrm{O}=\square_{5}
\end{aligned}
$$

for the invariant scalar product of real vector fields

$$
(C, B)=\int_{-\infty}^{+\infty} d^{4} \bar{x} \int_{0}^{2 \pi R} d s C_{A}(\bar{x}, s) B^{A}(\bar{x}, s) .
$$

This leads to

$$
\int d^{5} x \partial_{A}\left(C_{B}\left(\partial^{A} B^{B}\right)-\left(\partial^{A} C_{B}\right) B^{B}\right)=0
$$

which, taking into account the decrease of the fields at infinity in the $\bar{x}$ space (essentially faster than $1 / \sqrt{|\bar{x}|}$ ), becomes the basic boundary relation

$$
\int d^{4} \bar{x}\left[\left(\partial_{s} C_{A}\right) B^{A}-C_{A}\left(\partial_{s} B^{A}\right)\right]_{s=0}^{s=2 \pi R}=0 .
$$

In the next section, upon the Kaluza-Klein reduction, the boundary conditions for the reduced fields will be obtained from reduced boundary relations compatible with (30). 


\subsubsection{Basic boundary relations. Generalized case $(\lambda \neq 0)$}

In the generalized case, the equations of motion (14) are written

$$
\Omega_{B}^{A} B^{B}=-M^{2} B^{A}
$$

with the operator

$$
\Omega^{A}{ }_{B}=\square_{5} \delta_{B}^{A}+(\lambda-1) \partial^{A} \partial_{B} .
$$

In order for the eigenvalue $M^{2}$ to be real, the hermiticity of this operator is imposed

$$
(\Omega C, B)=(C, \Omega B)
$$

for the scalar product (28). This leads to the boundary relation

$$
\begin{aligned}
\int d^{4} \bar{x} & {\left[\left(C_{A}\left(\partial_{s} B^{A}\right)-\left(\partial_{s} C_{A}\right) B^{A}\right)\right.} \\
& \left.+(\lambda-1)\left(C_{S}\left(\partial_{A} B^{A}\right)-\left(\partial_{A} C^{A}\right) B_{S}\right)\right]_{0}^{2 \pi R}=0
\end{aligned}
$$

which generalizes (30), since for $\lambda=0, \partial_{A} B^{A}=0$ by (77)).

\subsection{Kaluza-Klein reduction. Mass towers. Boundary conditions}

\subsubsection{Kaluza-Klein reduction. Usual case $(\lambda=0)$}

We perform the following Kaluza-Klein reduction

$$
\begin{aligned}
B^{\mu}(\bar{x}, s) & =\sum_{r} B_{r}^{[V]}(s) \lambda_{r}^{\mu}(\bar{x}) \\
B^{5}(\bar{x}, s) & =\sum_{p} B_{p}^{[S]}(s) \phi_{p}(\bar{x})
\end{aligned}
$$

which breaks the $\mathrm{SO}(4,1)$ covariance and respects the $\mathrm{SO}(3,1)$ covariance in any brane (at fixed $s$ ).

The functions $B_{r}^{[V]}(s)$ and $B_{r}^{[S]}(s)$ (35), (36) are supposed to satisfy

$$
\begin{aligned}
& \partial_{s}^{2} B_{r}^{[V]}(s)=-\left(v_{r}^{[V]}\right)^{2} B_{r}^{[V]}(s) \\
& \partial_{s}^{2} B_{p}^{[S]}(s)=-\left(v_{p}^{[S]}\right)^{2} B_{p}^{[S]}(s)
\end{aligned}
$$


in such a way that the basic equations (8), applied for each $r$ (or $p$ ), reduce to

$$
\begin{aligned}
& \left(\square_{4}+\left(m_{r}^{[V]}\right)^{2}\right) \lambda_{r}^{\mu}(\bar{x})=0 \\
& \left(\square_{4}+\left(m_{p}^{[S]}\right)^{2}\right) \phi_{p}(\bar{x})=0 .
\end{aligned}
$$

The 4-dimensional masses for the 4-vector $\lambda_{r}^{\mu}(\bar{x})$ and for the 4-scalar $\phi_{p}(\bar{x})$ are then given by

$$
\begin{aligned}
& \left(m_{r}^{[V]}\right)^{2}=\left(v_{r}^{[V]}\right)^{2}+M^{2} \\
& \left(m_{p}^{[S]}\right)^{2}=\left(v_{p}^{[S]}\right)^{2}+M^{2} .
\end{aligned}
$$

The indices $r$ and $p$ in (35) and (36) refer respectively to the 4-dimensional masses squared $\left(m_{r}^{[V]}\right)^{2}$ and $\left(m_{p}^{[S]}\right)^{2}$ with by convention $\left(m_{r}^{[V]}\right)^{2}$ increasing with $r$ and $\left(m_{p}^{[S]}\right)^{2}$ increasing with $p$. Together they build up the $B^{A}$ tower.

Imposing the hermiticity (26) of the operator $\partial_{s}^{2}$ appearing in (37), (38) for the scalar product between functions of $s$

$$
(G, F)=\int_{s=0}^{2 \pi R} G(s) F(s) d s
$$

ensures the reality of its eigenvalues and so of the corresponding 4-dimensional masses squared (which could be negative). This leads to boundary relations

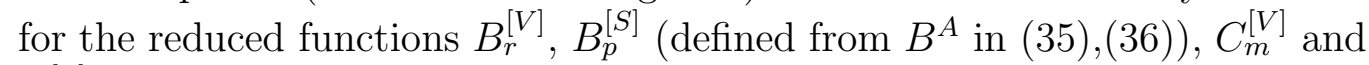
$C_{q}^{[S]}$ (defined similarly from $C^{A}$ )

$$
\begin{gathered}
{\left[\left(\partial_{s} C_{m}^{[V]}\right) B_{r}^{[V]}-C_{m}^{[V]}\left(\partial_{s} B_{r}^{[V]}\right)\right]_{0}^{2 \pi R}=0} \\
{\left[\left(\partial_{s} C_{q}^{[S]}\right) B_{p}^{[S]}-C_{q}^{[S]}\left(\partial_{s} B_{p}^{[S]}\right)\right]_{0}^{2 \pi R}=0 .}
\end{gathered}
$$

These boundary relations are compatible with the basic boundary relations (30).

Boundary conditions result from these boundary relations under the hypothesis that, on one side, all the reduced fields related to $B^{\mu}$ and $C^{\mu}$ live in some common domain in the Hilbert space and that, on the other side, all the 
reduced fields in $B^{5}$ and $C^{5}$ live in some, eventually different from the first one, common domain. Hence, the reduced fields are submitted to boundary conditions which must be independent of the index $r$ in (35), independent of the index $p$ in (36), but may depend on the $[V]$ or $[S]$ index.

Thus, for any field $B$ the boundary conditions have to be expressed by two linear homogeneous relations, independent of $r$, among the following quantities

$$
B_{r}^{[V]}(0), B_{r}^{[V]}(2 \pi R), \partial_{s} B_{r}^{[V]}(0), \partial_{s} B_{r}^{[V]}(2 \pi R)
$$

and similarly by two such relations, independent of $p$, among

$$
B_{p}^{[S]}(0), B_{p}^{[S]}(2 \pi R), \partial_{s} B_{p}^{[S]}(0), \partial_{s} B_{p}^{[S]}(2 \pi R) .
$$

For $B_{n}^{[S]}$, all the sets of allowed boundary conditions compatible with the boundary relations (44) are listed in Table (11). The boundary conditions for $B_{n}^{[V]}$ are identical to those for $B_{n}^{[S]}$ with the parameters $\left\{\alpha_{1}^{[S]}, \ldots\right\}$ replaced by $\left\{\alpha_{1}^{[V]}, \ldots\right\}$. Remark that all these boundary conditions are essentially the same as those allowed for a free real scalar field (see the first reference in [1]).

The eigenvalues $\left(v_{r}^{[V]}\right)^{2}$ of Eq.(37) corresponding to a chosen set of boundary conditions for $B_{r}^{[V]}$ define the corresponding Kaluza-Klein mass tower for $B^{\mu}$, and analogously the $\left(v_{p}^{[S]}\right)^{2}$ define the mass tower for $B^{S}$. The full $B^{A}$ tower is composed of all the vector $\left(m_{r}^{[V]}\right)^{2}$ and scalar $\left(m_{p}^{[S]}\right)^{2}$ squared masses (41) organized in such a way that $m_{\{n\}}^{2}$ increases with some index which we call $\{n\}$.

There are two possibilities. Either the same set of boundary conditions is imposed to $B_{r}^{[V]}$ and to $B_{p}^{[S]}$, in which case the corresponding spectra (37), (38) of eigenvalues coincide. Or different sets of boundary conditions are imposed to $B_{r}^{[V]}$ and to $B_{p}^{[S]}$, in which case the Kaluza-Klein tower is composed of the two corresponding spectra. This conforms to the idea that $\mathrm{SO}(4,1)$ is broken to $\mathrm{SO}(3,1)$ both by the compactification of the $s$ coordinate and by the Kaluza-Klein reduction (35)-(36). In general, for a given $m_{\{n\}}^{2}$, there are three possible situations

(1) a vector $\left(B_{\{n\}}^{[V]} \lambda_{\{n\}}^{\mu} \neq 0\right)$ and no scalar particle $\left(B_{\{n\}}^{[S]} \phi_{\{n\}}=0\right)$

(2) no vector $\left(B_{\{n\}}^{[V]} \lambda_{\{n\}}^{\mu}=0\right)$ and a scalar particle $\left(B_{\{n\}}^{[S]} \phi_{\{n\}} \neq 0\right)$

(3) a vector $\left(B_{\{n\}}^{[V]} \lambda_{\{n\}}^{\mu} \neq 0\right)$ and a scalar particle $\left(B_{\{n\}}^{[S]} \phi_{\{n\}} \neq 0\right)$.

Depending on $\{n\}$, any of these three situations may occur. 


\subsubsection{Kaluza-Klein reduction. Generalized case $(\lambda \neq 0)$}

In the generalized case $\lambda \neq 0$, starting from the equations of motion (16) or (23) -(25) for the physical fields $B^{\prime} A$ and $B^{\prime}$, we perform the following Kaluza-Klein reduction

$$
\begin{aligned}
B^{\prime \mu}(\bar{x}, s) & =\sum_{r} B_{r}^{{ }^{\prime}[V]}(s) \lambda_{r}^{\prime \mu}(\bar{x}) \\
B^{\prime 5}(\bar{x}, s) & =\sum_{p} B_{p}^{\prime[S]}(s) \phi_{p}^{\prime}(\bar{x}) \\
B^{\prime}(\bar{x}, s) & =\sum_{v} B_{v}^{\prime}(s) \psi_{v}^{\prime}(\bar{x}) .
\end{aligned}
$$

Imposing the hermiticity of $\partial_{s}^{2}$ and following the usual procedure, one obviously finds that the reduced fields $B_{r}^{\prime[V]}(s), B_{p}^{\prime[S]}$ and $B_{v}^{\prime}(s)$ must satisfy boundary relations of the form (43)-(44)

$$
\begin{aligned}
{\left[\left(\partial_{s} C_{m}^{\prime[V]}\right) B_{r}^{\prime[V]}-C_{m}^{\prime[V]}\left(\partial_{s} B_{r}^{\prime[V]}\right)\right]_{0}^{2 \pi R}=0 } \\
{\left[\left(\partial_{s} C_{q}^{\prime[S]}\right) B_{p}^{\prime[S]}-C_{q}^{\prime[S]}\left(\partial_{s} B_{p}^{\prime[S]}\right)\right]_{0}^{2 \pi R}=0 } \\
{\left[\left(\partial_{s} C_{v}^{\prime}\right) B_{w}^{\prime}-C_{v}^{\prime}\left(\partial_{s} B_{w}^{\prime}\right)\right]_{0}^{2 \pi R}=0 . }
\end{aligned}
$$

The derived boundary conditions must be the same for all the reduced components of each of the fields. In other words, the boundary conditions in terms of the fields $B_{r}^{[[V]}(s), B_{p}^{\prime[S]}$ and $B_{v}^{\prime}(s)$ evaluated at $s=0$ and $s=2 \pi R$ (see the discussion of (45)) must be independent of the indices $r, p$ and $v$ respectively.

Refering to (17), it is natural, in order to obtain definite boundary conditions for $B^{A}$, to impose the same boundary conditions for $B^{\prime} A$ and $\partial^{A} B^{\prime}$

$$
\begin{array}{ccc}
B_{r}^{\prime[V]}(s) & \text { same boundary conditions as } & B_{v}^{\prime}(s) \\
B_{r}^{[[S]}(s) & \text { same boundary conditions as } & \partial_{s} B_{v}^{\prime}(s)
\end{array}
$$

independently of $r$ and $v$. 


\subsubsection{Physical implication $(\lambda \neq 0)$}

Since $B_{r}^{\prime[V]}$ and $B_{v}^{\prime}$ are submitted to the same boundary conditions (53), their eigenvalue spectrum $v_{n}^{2}$ are identical. Hence, the scalar and vector mass towers of the $\lambda \neq 0$ case are the scalar and vector mass towers of the $\lambda=0$ case accompanied by a new scalar mass tower (with fields $\psi_{p}^{\prime}(\bar{x})$ ) with all their masses, according to (16), shifted by

$$
\text { Square mass shift }=\left(m_{n}^{[\psi]}\right)^{2}-\left(m_{n}^{[V]}\right)^{2}=\frac{1-\lambda}{\lambda} M^{2} .
$$

\subsection{Gauge condition}

\subsubsection{Gauge condition. Usual case $(\lambda=0)$}

The Lorenz condition (77), restricted to one $\{n\}$, reduces to

$$
B_{\{n\}}^{[V]}\left(\partial_{\mu} \lambda_{\{n\}}^{\mu}\right)+\left(\partial_{s} B_{\{n\}}^{[S]}\right) \phi_{\{n\}}=0 .
$$

In order to have a non-zero vector and/or a non-zero scalar part $\{n\}$ in the tower (not both zero), this equation implies in general (taking into account an arbitrary normalisation) one of the following four distinct physical cases

Generic Case 1: $B_{\{n\}}^{[V]} \neq 0, \partial_{s} B_{\{n\}}^{[S]}=B_{\{n\}}^{[V]},\left\{\begin{array}{l}\partial_{\mu} \lambda_{\{n\}}^{\mu}+\phi_{\{n\}}=0 \\ \phi_{\{n\}} \neq 0\end{array}\right.$

Generic Case 2: $B_{\{n\}}^{[V]} \neq 0$, no scalar $\quad, \quad \partial_{\mu} \lambda_{\{n\}}^{\mu}=0$

Special Case 1: $B_{\{n\}}^{[V]} \neq 0, B_{\{n\}}^{[S]}=1 \quad,\left\{\begin{array}{l}\partial_{\mu} \lambda_{\{n\}}^{\mu}=0 \\ \phi_{\{n\}} \neq 0\end{array}\right.$

Special Case 2: no vector, $B_{\{n\}}^{[S]}=1 \quad, \quad \phi_{\{n\}} \neq 0$.

From these equations, the physical content of the tower at level $\{n\}$ can be easily deduced for every case (57)-(60). In general, the Generic Cases are related to tower states while the Special Cases correspond (within a tower) to specific states characterized by $v_{\{n\}}^{2}=0$.

- Generic Case 1 when $m_{\{n\}}^{2}=M^{2}+v_{\{n\}}^{2} \neq 0$ (see (57)). For any such $\{n\}$, there is a 4-dimensional vector field $A_{\{n\}}^{\mu}$ and a 4-dimensional 
scalar field $\phi_{\{n\}}$ given by

Generic Case 1: $\left\{\begin{array}{c}\text { vector field } A_{\{n\}}^{\mu} \\ \text { scalar field } \phi_{\{n\}} \\ m_{\{n\}}^{2} \neq 0\end{array}\left\{\begin{array}{c}A_{\{n\}}^{\mu}=\lambda_{\{n\}}^{\mu}-\frac{\partial^{\mu} \phi_{\{n\}}}{m_{\{n\}}^{2}} \\ \left(\square_{4}+m_{\{n\}}^{2}\right) \phi_{\{n\}}=0 \\ \left(\square_{4}+m_{\{n\}}^{2}\right) A_{\{n\}}^{\mu}=0 \\ \partial_{\mu} A_{\{n\}}^{\mu}=0\end{array}\right.\right.$.

Note that the vector $A_{\{n\}}^{\mu}$ is a true physical vector field, which satisfies as expected the 4-dimensional Lorenz condition.

- Generic Subcase 1 when, for a given $\{n\}, m_{\{n\}}^{2}=M^{2}+v_{\{n\}}^{2}=0$. There is then a 4-dimensional massless scalar field $\phi_{\{n\}}$, and a 4-dimensional massless vector field $A_{\{n\}}^{\mu}$ which satisfy

Generic Subcase 1: $\left\{\begin{array}{c}\text { vector field } A_{\{n\}}^{\mu} \\ \text { scalar field } \phi_{\{n\}}^{A} \\ \left(m_{\{n\}}^{A}\right)^{2}=\left(m_{\{n\}}^{\phi}\right)^{2}=0\end{array} \quad\left\{\begin{array}{l}A_{\{n\}}^{\mu}=\lambda_{\{n\}}^{\mu} \\ \square_{4} A_{\{n\}}^{\mu}=0 \\ \square_{4} \phi_{\{n\}}=0 \\ \partial_{\mu} A_{\{n\}}^{\mu}=-\phi_{\{n\}}\end{array}\right.\right.$

The equations for $A_{\{n\}}^{\mu}$ and $\phi_{\{n\}}$ in four dimensions are of the same form as the equations (23)-(25) for respectively $B^{\prime} A$ and $B^{\prime}$ in five dimensions for $\lambda=-1$. They belong to a generalized 4-dimensional case with $\lambda \neq 0$. Refering to the discussion following (25), we conclude that $\phi_{\{n\}}$ describes a scalar massless particle in the brane and that $A_{\{n\}}^{\mu}$ describes a particle whose 4-dimensional divergence is proportional to $\phi_{\{n\}}$, whose longitudinal part can be removed by a suitable partial gauge transformation and whose two truly transverse degrees of freedom describe a physical massless vector field in the brane.

- Generic Case 2 with $m_{\{n\}}^{2}=M^{2}+v_{\{n\}}^{2}$ (see (58)). For any $\{n\}$ with $m_{\{n\}}^{2}=v_{\{n\}}^{2}+M^{2}$, there is a true 4-dimensional vector field $A_{\{n\}}^{\mu}$ and no scalar field.

Generic Case 2: $\left\{\begin{array}{c}\text { vector field } A_{\{n\}}^{\mu} \\ \text { no scalar field } \\ m_{\{n\}}^{2}=v_{\{n\}}^{2}+M^{2}\end{array}\left\{\begin{array}{l}A_{\{n\}}^{\mu}=\lambda_{\{n\}}^{\mu} \\ \left(\square_{4}+m_{\{n\}}^{2}\right) A_{\{n\}}^{\mu}=0 \\ \partial_{\mu} A_{\{n\}}^{\mu}=0 \\ \text { no } \phi_{\{n\}}\end{array}\right.\right.$. 
- Special Case 1 (59). Both a 4-dimensional vector $A_{\{n\}}^{\mu}$ and a 4-dimensional scalar $\phi_{\{n\}}$ field are present in the brane and, from (38), $v_{\{n\}}^{2}=0$. Their mass squared is equal to the bulk mass squared.

$$
\text { Special Case 1: }\left\{\begin{array} { c } 
{ \text { vector field } A _ { \{ n \} } ^ { \mu } } \\
{ \text { scalar field } \phi _ { \{ n \} } } \\
{ m _ { \{ n \} } ^ { 2 } = M ^ { 2 } }
\end{array} \left\{\begin{array}{l}
A_{\{n\}}^{\mu}=\lambda_{\{n\}}^{\mu} \\
\left(\square_{4}+M^{2}\right) \phi_{\{n\}}=0 \\
\left(\square_{4}+M^{2}\right) A_{\{n\}}^{\mu}=0 \\
\partial_{\mu} A_{\{n\}}^{\mu}=0
\end{array} .\right.\right.
$$

- Special Case 2 (60) . There is a no 4-dimensional vector state but a lonely 4-dimensional scalar state $\phi_{\{n\}}$ is present and, from (38), $v_{\{n\}}^{2}=0$. This means that the scalar has a brane mass squared equal to the bulk mass squared.

$$
\text { Special Case } 2:\left\{\begin{array} { c } 
{ \text { no vector field } } \\
{ \text { scalar field } \phi _ { \{ n \} } } \\
{ m _ { \{ n \} } ^ { 2 } = M ^ { 2 } }
\end{array} \left\{\begin{array}{l}
\left(\square_{4}+M^{2}\right) \phi_{\{n\}}=0 \\
\text { no } \lambda_{\{n\}}^{\mu}
\end{array}\right.\right.
$$

\subsubsection{Gauge condition. Generalized case $(\lambda \neq 0)$}

Remembering the discussion concerning Generic Case 1 (57) and Generic Case 2 (58) and taking into account Eq.(17), (53), (54), we have the general possibilities

Generalized Generic Case 1 : same BC for $B_{r}^{\prime[V]}, \partial_{s} B_{r}^{\prime[S]}$ and $B_{v}^{\prime}$

Generalized Generic Case $2: B_{r}^{\prime[S]}=0$, same BC for $B_{r}^{\prime[V]}$ and $B_{v}^{\prime}$.

The discussion of the Lorenz condition (ㄷ6) (for $B^{\prime[V]}$ and $B^{\prime[S]}$ ) and of the the Extended Special Cases 1 and 2 follow the same line as in the $\lambda=0$ case with obvious changes. From here on, we concentrate on the usual case $(\lambda=0)$.

\subsection{Final sets of allowed boundary conditions}

The sets of boundary conditions (abbreviated BC in this subsection) for $B_{\{n\}}^{[S]}$ and $B_{\{n\}}^{[V]}$ (see Table (1) ) have to be made compatible with each other, taking into account the eigenvalue equations (37), (38) and in particular the 
Lorenz condition (56). The results, as obtained in App.(A), are summarized hereafter. The generic cases are related to $\mathrm{BC}$ which lead to towers composed of an infinite number of states. The special cases are related to BC which lead to the presence of specific states in the towers.

\section{- Generic Case 1}

In the Generic Case 1 (57), the allowed sets of $\mathrm{BC}$ for $B_{n}^{[V]}$ and $B_{n}^{[S]}$ are given in the following Table

Sets G1 $\left\{\begin{array}{|c|c|c|c|c|}\hline \text { Set } & & & & \\ \hline \hline \text { G1a } & \begin{array}{c}B_{n}^{[V]}(2 \pi R) \\ = \\ \alpha B_{n}^{[V]}(0)\end{array} & \begin{array}{c}\partial_{s} B_{n}^{[V]}(2 \pi R) \\ = \\ \alpha\end{array} \partial_{s} B_{n}^{[V]}(0) & \begin{array}{c}B_{n}^{[S]}(2 \pi R) \\ = \\ \frac{1}{\alpha} B_{n}^{[S]}(0)\end{array} & \begin{array}{c}\partial_{s} B_{n}^{[S]}(2 \pi R) \\ = \\ \alpha \partial_{s} B_{n}^{[S]}(0)\end{array} \\ \hline \text { G1b } & B_{n}^{[V]}(0)=0 & B_{n}^{[V]}(2 \pi R)=0 & \partial_{s} B_{n}^{[S]}(0)=0 & \partial_{s} B_{n}^{[S]}(2 \pi R)=0 \\ \hline \text { G1c } & \partial_{s} B_{n}^{[V]}(0)=0 & \partial_{s} B_{n}^{[V]}(2 \pi R)=0 & B_{n}^{[S]}(0)=0 & B_{n}^{[S]}(2 \pi R)=0 \\ \hline \text { G1d } & B_{n}^{[V]}(0)=0 & \partial_{s} B_{n}^{[V]}(2 \pi R)=0 & \partial_{s} B_{n}^{[S]}(0)=0 & B_{n}^{[S]}(2 \pi R)=0 \\ \hline \text { G1e } & \partial_{s} B_{n}^{[V]}(0)=0 & B_{n}^{[V]}(2 \pi R)=0 & B_{n}^{[S]}(0)=0 & \partial_{s} B_{n}^{[S]}(2 \pi R)=0 \\ \hline\end{array}\right.$

\section{- Generic Case 2}

In the Generic Case 2 (58), the allowed sets of BC are

$$
\text { Sets G2 }\left\{\begin{array}{ll}
B_{\{n\}}^{[S]}=0 & \begin{array}{l}
\text { special solution compatible with } \\
\text { any set of BC of Table (1) }
\end{array} \\
\text { for } B_{\{n\}}^{[V]} \neq 0 & \text { any set of BC of Table (1) }
\end{array} .\right.
$$

\section{- Special Case 1}

In the Special Case 1 (59), the allowed sets of BC are

$$
\text { Set S1a }\left\{\begin{array}{cc}
B_{\{n\}}^{[S]}=1 & \begin{array}{l}
\text { special solution of a subset } \\
\text { of the set A1 of Table (1) }
\end{array} \\
& \left\{\begin{array}{l}
B_{\{n\}}^{[S]}(2 \pi R)=B_{\{n\}}^{[S]}(0)+\alpha_{2}^{[S]} \\
\partial_{s} B_{\{n\}}^{[S]}(2 \pi R)=\partial_{s} B_{\{n\}}^{[S]}(0)
\end{array}\right. \\
& \begin{array}{l}
{[S]} \\
\text { for } \left.B_{\{n\}}^{[V]} \neq 0\right)
\end{array}
\end{array}\right.
$$


Set S1b $\left\{\begin{array}{cc}B_{\{n\}}^{[S]}=1 & \begin{array}{c}\text { special solution of a subset } \\ \text { of the set A2 of Table (1) }\end{array} \\ & \left\{\begin{array}{c}\partial_{s} B_{\{n\}}^{[S]}(0)=0 \\ \partial_{s} B_{\{n\}}^{[S]}(2 \pi R)=0\end{array}\right. \\ \text { for } B_{\{n\}}^{[V]} \neq 0 \text { any set of BC of Table (11) }\end{array}\right.$

\section{- Special Case 2}

In the Special Case 2 (60), the allowed sets of BC are

$$
\begin{aligned}
& \text { Set S2a }\left\{\begin{array}{cl}
B_{\{n\}}^{[S]}=1 & \begin{array}{c}
\text { special solution of the subset } \\
\text { of the set A1 of Table (1) }
\end{array} \\
& \left\{\begin{array}{c}
B_{\{n\}}^{[S]}(2 \pi R)=B_{\{n\}}^{[S]}(0)+\alpha_{2}^{[S]} \partial_{s} B_{\{n\}}^{[S]}(0) \\
\partial_{s} B_{\{n\}}^{[S]}(2 \pi R)=\partial_{s} B_{\{n\}}^{[S]}(0)
\end{array}\right. \\
B_{\{n\}}^{[V]}=0 \begin{array}{l}
\text { special solution of any } \\
\text { set of BC of Table (1) }
\end{array}
\end{array}\right. \\
& \text { Set S2b }\left\{\begin{array}{cc}
B_{\{n\}}^{[S]}=1 & \begin{array}{c}
\text { special solution of the subset } \\
\text { of the set A2 of Table (1) }
\end{array} \\
& \left\{\begin{array}{c}
\partial_{s} B_{\{n\}}^{[S]}(0)=0 \\
\partial_{s} B_{\{n\}}^{[S]}(2 \pi R)=0
\end{array}\right. \\
B_{\{n\}}^{[V]}=0 & \begin{array}{l}
\text { special solution of any } \\
\text { set of BC of Table (11) }
\end{array}
\end{array}\right.
\end{aligned}
$$

\section{Physical considerations}

\subsection{Physical discussion of the boundary conditions. Clo- sure to a circle}

If one looks at the boundary conditions of Table (11), one sees that there are two very different situations. For the Sets A2, A3, A4, A5, the boundary conditions are local. The values of the fields are related at the same point, either at $s=0$ or at $s=2 \pi R$. The boundary conditions A1 are of a rather different kind as they connect values of the fields evaluated at these two different points of the $s$ domain. In this latter case, the field explores in fact its full domain at once. This is tantamount to action at a distance or to non 
locality. In the third article of [1], we noted that this was not in contradiction with quantum mechanics.

Under the A1 boundary conditions with $\alpha_{2}=\alpha_{3}=0$, the strip can be closed into a circle by identifying the points $s=0$ and $s=2 \pi R$. With $\alpha_{1}=\alpha_{4}=1$ this leads to the periodic boundary conditions, and with $\alpha_{1}=$ $\alpha_{4}=-1$ to the antiperiodic boundary conditions. They are both of local type.

\subsection{Mass considerations}

For completeness, we will give, in the next subsection, a description of all the allowed mass equations from which the mass towers are derived. We remind that we are here restricted to a five dimensional flat space with a compactified fifth coordinate. In general, an evaluation of the low lying mass eigenvalues give values of the form $1 / R$ multiplied by a factor of order 1 . For mass eigenvalues of the order of $1 \mathrm{TeV}$, this requires $R$ to be of the order $10^{-16} \mathrm{~cm}$.

This value is far away from the $R \approx 10^{10} \mathrm{~cm}$ originating from the relation

$$
M_{P l}^{2} \approx V_{\{n\}} M_{*}^{n+2}
$$

between the Planck mass $M_{P l}$, the fundamental scale $M_{*}$ and the volume $V_{5}=$ $(2 \pi R)^{5}$ under the hypothesis that Gauss law is valid in the five dimensional space. This idea has been discussed at length in the litterature [3], but the results are still under investigation. As is well-known the problem has been cured elegantly by Randall and Sundrum [4] by the use of warped spaces (see also [5]) as will be discussed in a subsequent publication.

\subsection{Mass considerations. Experimental aspects}

The presence or absence of scalars states in the Kalauza-Klein towers can roughly be summarized as follows

(1) $\lambda \neq 0$, Generic Case 1 (61)

Accompanying the 4-dimensional vector tower there are two 4-dimensional scalar towers. The masses in one of the scalar tower are identical to the masses in the vector tower while the masses in the second scalar tower are shifted from the masses in the vector tower by the fixed amount (55). 
(2) $\lambda \neq 0$, Generic Case 2 (63)

Accompanying the 4-dimensional vector tower there is one 4-dimensional scalar tower. The masses in the scalar tower are shifted from the masses in the vector tower by the fixed amount (55).

(3) $\lambda=0$, Generic Case 1 (61)

Accompanying the 4-dimensional vector tower there is one 4-dimensional scalar tower. The masses in the scalar tower are identical to the masses in the vector tower.

(4) $\lambda=0$, Generic Case 2 (63)

There is one 4-dimensional vector tower and no 4-dimensional scalar tower.

An examination of the masses of the known bosons as given by the Particle Data Group [6], reveals at first sight that, to any set of observed vector (or pseudo-vector) particles, there is no sign of a related set of scalar (or pseudoscalar) particles either with identical masses or with masses squared shifted by a fixed amount.

Hence, if a Kaluza-Klein tower of vector mesons exists and some of its low lying states already appear in the Particle Data Group tables, the theory must belong to the case (4) above i.e. must correspond to a Lagrangian with $\lambda=0$ while the boundary conditions must belong to the Generic Case 2 .

\section{Towers}

In this section, we give the main properties of the vector and related scalar towers which follow from specific choices of allowed boundary conditions ( and $(\underline{69})$ ). The detailed towers are obtained by following the procedure used in the first article of [1]

\subsection{Towers in the Generic Case G1a}

The general solution of $B_{\{n\}}^{[S]}(s)$ for $v_{n}^{2}>0$ is a superposition of sine and cosine functions (see (75) ). For $v_{n}^{2}<0$ it is a superposition of hyperbolic sine and cosine functions while for $v_{n}=0$ it is a linear function in $s$. It is easy to see that the hyperbolic solution is incompatible with the G1a boundary 
conditions in Table (68) for any $\alpha$, while the linear solution can only be a constant and is compatible with $\alpha=1$ only. We thus focuss of the sine and cosine solution.

Writing the general solution $B_{\{n\}}^{[S]}(s)$ of (38) (taking $v_{\{n\}}>0$ ) as

$$
B_{\{n\}}^{[S]}(s)=\sigma_{\{n\}}^{[S]} \sin \left(v_{\{n\}} s\right)+\tau_{\{n\}}^{[S]} \cos \left(v_{\{n\}} s\right),
$$

with $B_{\{n\}}^{[V]}(s)=\partial_{s} B_{\{n\}}^{[S]}(s)$, the set of boundary conditions G1a (하) leads to the eigenvalue equation for $v_{\{n\}}$

$$
\cos \left(2 \pi v_{\{n\}} R\right)=\frac{2}{\alpha+\frac{1}{\alpha}}
$$

with

$$
\sin \left(2 \pi v_{\{n\}} R\right)=\epsilon \frac{\alpha^{2}-1}{\alpha^{2}+1} \quad, \quad \epsilon^{2}=1
$$

If $\alpha^{2} \neq 1$, the field parameters $\sigma_{\{n\}}^{[S]}$ and $\tau_{\{n\}}^{[S]}$ are related by

$$
\tau_{\{n\}}^{[S]}=-\epsilon \alpha \sigma_{\{n\}}^{[S]}
$$

while if $\alpha^{2}=1$ they are arbitrary.

Calling

$$
w=\frac{1}{\pi} \arccos \left(\frac{2}{\alpha+\frac{1}{\alpha}}\right) \quad, \quad 0 \leq w \leq 1
$$

the mass tower $m_{\{n\}}^{2}$ is composed of the $\epsilon=+1$ and $\epsilon=-1$ intertwined branches

$$
\begin{array}{ccc}
n=2 p+1 \quad, \quad & m_{\{2 p+1\}}^{2}=M^{2}+\frac{(2 p+w)^{2}}{4 R^{2}} \quad, \quad p \geq 0 \\
n=2 p \quad, \quad m_{\{2 p\}}^{2}=M^{2}+\frac{(2 p-w)^{2}}{4 R^{2}} \quad, \quad p \geq 1 .
\end{array}
$$

Note that the index $\{n\}$ is defined in such a way that the first state in the tower corresponds to $n=1$ and that the masses are ordered in such a way that they increase with $n$. Remark also that for $\alpha= \pm 1$, every state in the tower is twice degenerate except for $\alpha=1$ where the first state with $m_{\{1\}}^{2}$ is single. Indeed, for $\alpha=1(w=0)$ one has $m_{\{2 q\}}^{2}=m_{\{2 q+1\}}^{2}, q \geq 0$ and for $\alpha=-1(w=1)$ one has $m_{\{2 q+1\}}^{2}=m_{\{2(q+1)\}}^{2}, q>0$. 
The only possibility for having $v_{\{1\}}=0$ is a constant $B_{\{n\}}^{[S]}(s)$. It implies $\alpha=1$ and corresponds to the Special Case 1 (59) or Special Case 2 (60) (see (64) or (65) ).

The lowest mass squared in the tower (41) is always larger than the bulk mass squared $M^{2}$ if $\alpha \neq 1$ and equal to it if $\alpha=1$. Note that the bulk mass squared can a priori be negative which means that the original five-dimensional field is tachyonic. This could lead, at the bottom of the tower, to the appearance of one or more four-dimensional tachyons. A zero mass state (eventually twice degenerate for $\alpha^{2}=1$ ) can occur provided the boundary parameter $\alpha$ is such that $M^{2}$ equals $-v_{\{n\}}^{2}$ i.e. the negative of one of the eigenvalues.

\subsection{Towers in the Generic Case G1b}

The boundary conditions in the Case G1b of (68), when imposed to the sine and cosine solution (75) for $B_{\{n\}}^{[S]}(s)\left(v_{\{n\}}>0\right)$, lead to the well-known regular tower

$$
v_{\{n\}}=\frac{n}{2 R} \quad \text { for } n=1,2, \ldots
$$

as the solutions of the equation

$$
\sin \left(2 \pi R v_{\{n\}}\right)=0
$$

There is no hyperbolic sine or cosine solution $\left(v_{\{n\}}^{2}<0\right)$. The case $v_{\{n\}}=0$,

corresponding to a linear $B_{\{n\}}^{[S]}(s)$, leads to a constant field, say $B_{\{n\}}^{[S]}(s)=1$ by normalisation. This corresponds to the lowest state $(n=0)$ in the tower which is of the (59) form. The resulting values of the masses squared of all the states in the tower are finally

$$
m_{\{n\}}^{2}=M^{2}+\left(\frac{n}{2 R}\right)^{2} \quad \text { for } n=0,1, \ldots
$$

\subsection{Towers in the Generic Case G1c}

Analogously to the preceding G1b case, the boundary conditions in the Case G1c of (68) lead for $B_{\{n\}}^{[S]}(s)$ to the sine and cosine solution when $v_{\{n\}}^{2}>0$ but neither to a constant solution for $v_{\{n\}}=0$ nor to a hyperbolic solution for $v_{\{n\}}^{2}<0$. The same regular tower (83) results except that the $n=0$ state is absent. 


\subsection{Towers in the Generic Cases G1d, G1e}

Analogously to the preceeding G1b, G1c cases, the boundary conditions in the Case G1d and G1e of (68) lead to the sine and cosine solution for $v_{\{n\}}^{2}>0$ but neither to a constant solution for $v_{\{n\}}^{2}=0$ nor to a hyperbolic solution for $v_{\{n\}}^{2}<0$. The $v_{\{n\}}>0$ must satisfy the equation

$$
\cos \left(2 \pi R v_{\{n\}}\right)=0
$$

and the masses squared of the states in the tower are

$$
m_{\{n\}}^{2}=M^{2}+\frac{1}{4 R^{2}}\left(n+\frac{1}{2}\right)^{2} \quad \text { for } n=0,1, \ldots .
$$

\subsection{Towers in the Generic Case G2}

Since the $B_{\{n\}}^{[V]}$ are allowed to satisfy any set of boundary conditions (69) as summarized in Table (11) (with $[P]$ replaced by $[V]$ ) and since $B_{\{n\}}^{[S]}=0$, there are only vector towers and no corresponding scalar towers. The allowed towers are summarized in Table (2). They are in complete correspondance with the towers obtained for scalar states in a flat space (see the first article in [1]). In general, one finds that the masses in the towers are derived from equations involving trigonometric functions and polynomials. These equations depend in a specific way on the parameters defining the boundary conditions and lead to less regular towers than the usual linear forms (83), (85) which were originally associated to Kaluza-Klein towers.

\section{Conclusions}

The occurence of Kaluza-Klein towers for vector fields propagating in a five dimensional compactified flat space might be interesting to look at, at high energies. In this paper, we have analyzed and presented the results of a careful study of all the sets of allowed boundary conditions which follow from the physical requirement of hermiticity (symmetry or self-adjointness) of the operators whose eigenvalues are related to the Kaluza-Klein mass tower states, as seen in a 4-brane.

Our approach starts from the most general free Lagrangian quadratic in the bulk vector field and its derivatives. This Lagrangian depends on two 
free parameters, the bulk mass $M$ and one extra parameter $\lambda$ (see Eq.(2) and Eq.(4)). The sets of allowed boundary conditions are restricted by the all important Lorenz 5-dimensional gauge condition.

In the case of the generalized Lagrangian $(\lambda \neq 0)$, there appears, for any set of boundary conditions and apart from the main tower constituted by vector states (with possibly an associated tower of scalar states with the same mass spectrum), an extra tower of scalar particles with masses squared which differ from the main tower masses squared by a universal additive term $(1-\lambda) M^{2} / \lambda$ which does not depend on the parameters chosen for the boundary conditions.

For the main tower, there are essentially two distinct situations. In the first one, the four-dimensional vector tower is associated to a fourdimensional scalar tower with coinciding masses. Their spectrum is simple and regular (see (80), (83) and (85) ). In the second situation, there is no associated scalar tower. The allowed vector mass spectra are much richer (see Tables (2), (3), (41)) and similar to the spectra found for the scalar and spinor towers (see the first and fourth articles of [1]).

It may be too naive to try to associate the towers for vector states that we have obtained from a flat five-dimensional compactified space to experimentally existing particle masses (3.3). Indeed, as is well-known, the mass scale given by the parameter $1 / R$, which should be of the order of Tev for a flat space, suffers from severe defects (3.2). Randall and Sundrum [4] elegantly cured this problem by the consideration of warped spaces with an exponential behavior of the metric in the extra dimension. We intend to address this issue in a forthcoming article.

Regarding the photon, it is believed that it has no associated massless scalar partner, as an axion like particle [7] has up to now escaped observation. If it is then a low lying state of a vector tower, boundary conditions of the Generic Case 2 type must apply. Moreover, the zero mass of the photon would require very precise relations (fine tuning!) between the free parameters, including the bulk mass.

Acknowledgment: The authors would like to thank Professor David Fairlie for a discussion about general solutions of the equations of motion and Professor Nicolas Boulanger for discussions in relation with particles of higher spins. 


\section{References}

[1] F. Grard, J. Nuyts, Phys. Rev. D 74, 124013 (2006), hep-th/0607246.

F. Grard, J. Nuyts, Phys. Rev. D 76, 124022 (2007), hep-th/0707.4562.

F. Grard, J. Nuyts, Nuc. Phys. B 811, 123-154 (2009), http://dx.doi.org/10.1016/j.nuclphysb.2008.11.016 hep-th/0712.3692.

F. Grard, J. Nuyts, Phys. Rev. D 78, 024020 (2008), hep-th/0803.1741.

F. Grard, J. Nuyts, Phys. Rev. D 79, 064019 (2009), hep-th/0812.4304.

[2] Kaluza,T., Sitzungsber. Preuss. Akad. Wiss. Berlin. (Math. Phys.), K1, 966-972 (1921). Klein,O., Z. Phys. 37, 895-906 (1926).

[3] Arkhani-Ahmed, N., Dimopoulos, S., Dvali, G., Phys. Lett., B429, 263 (1998), hep-ph/9803315, SLAC-PUB-7769, SU-ITP-98/13.

[4] L. Randall, R. Sundrum, Phys. Rev. Lett. 83, 3370 (1999), hep-ph/9905221.

L. Randall, R. Sundrum, Phys. Rev. Lett. 83, 4690 (1999), hep-th/9906064.

[5] Gherghetta, T., Pomarol, A., Nucl. Phys. B 586, 141 (2000).

[6] C. Amsler et al. (Particle Data Group), Physics Letters B 667, 1 (2008) and (2009) partial update for the (2010) edition.

[7] S. Weinberg, Phys. Rev. Letters, 40, 223 (1978),

F. Wilczek, Phys. Rev. Letters, 40, 279 (1978). 


\section{A Discussion of the joined boundary condi- tions for $B_{\{n\}}^{[V]}$ and $B_{\{n\}}^{[S]}$ resulting from the gauge conditions}

The a priori independent allowed sets of boundary conditions for $B_{\{n\}}^{[S]}$ and $B_{\{n\}}^{[V]}$ are given in Table (11). The gauge conditions (56) imply that these functions and hence their respective boundary conditions are related (see (57) $-(\underline{601})$ ).

\section{A.1 Generic Case 1}

In the Generic Case 1 (57), the functions $B_{\{n\}}^{[V]}$ and $B_{\{n\}}^{[S]}$ are related by

$$
\partial_{s} B_{\{n\}}^{[S]}=B_{\{n\}}^{[V]}
$$

This implies the following compatibility conditions

- Set A1 for $B_{\{n\}}^{[V]}$ (see Table (1i)).

If the boundary conditions for $B_{\{n\}}^{[V]}$ are of the form A1

$$
\begin{aligned}
B_{\{n\}}^{[V]}(2 \pi R)= & \alpha_{1}^{[V]} B_{\{n\}}^{[V]}(0)+\alpha_{2}^{[V]} \partial_{s} B_{\{n\}}^{[V]}(0) \\
\partial_{s} B_{\{n\}}^{[V]}(2 \pi R)= & \alpha_{3}^{[V]} B_{\{n\}}^{[V]}(0)+\alpha_{4}^{[V]} \partial_{s} B_{\{n\}}^{[V]}(0) \\
& \alpha_{1}^{[V]} \alpha_{4}^{[V]}-\alpha_{2}^{[V]} \alpha_{3}^{[V]}=1,
\end{aligned}
$$

those deduced naively from (ㅎ6) for $B_{\{n\}}^{[S]}$, taking into account ( also boundary conditions of the form A1 for $B_{\{n\}}^{[S]}$ with

$$
\begin{aligned}
\alpha_{1}^{[S]} & =\alpha_{4}^{[V]} \\
\alpha_{2}^{[S]} & =-\frac{\alpha_{3}^{[V]}}{v_{\{n\}}^{2}} \\
\alpha_{3}^{[S]} & =-v_{\{n\}}^{2} \alpha_{2}^{[V]} \\
\alpha_{4}^{[S]} & =\alpha_{1}^{[V]} .
\end{aligned}
$$


Since the boundary parameters must be independent of $\{n\}$, and taking into account that $\alpha_{1}^{[S]} \alpha_{4}^{[S]}-\alpha_{2}^{[S]} \alpha_{3}^{[S]}$ must be equal to 1 , the values of all the parameters are given in terms of one parameter only, say $\alpha$

$$
\begin{aligned}
& \alpha_{2}^{[S]}=\alpha_{3}^{[S]}=\alpha_{2}^{[V]}=\alpha_{3}^{[V]}=0 \\
& \alpha_{1}^{[V]}=\alpha, \quad \alpha_{4}^{[V]}=\frac{1}{\alpha}, \quad \alpha_{1}^{[S]}=\frac{1}{\alpha}, \quad \alpha_{4}^{[S]}=\alpha
\end{aligned}
$$

leading to the set G1a in (68).

- Set A2 for $B_{\{n\}}^{[V]}$ (see Table (10)).

Applying the same reasoning as for the set A1, one finds that the only allowed case A2 for $B_{\{n\}}^{[V]}$ is with $\rho_{1}^{[V]}=0$ and $\rho_{2}^{[V]}=0$. It implies the case A5 for $B_{\{n\}}^{[S]}$. The set G1c in (68) follows.

- Set A3 for $B_{\{n\}}^{[V]}$ (see Table (10)).

Applying the same reasoning as for the set A1, one finds that the only allowed case A3 for $B_{\{n\}}^{[V]}$ is with $\kappa^{[V]}=0$. It implies the case A4 for $B_{\{n\}}^{[S]}$ with $\zeta^{[S]}=0$. The set G1d in (하) follows.

- Set A4 for $B_{\{n\}}^{[V]}$ (see Table (11)).

Applying the same reasoning as for the set A1, one finds that the only allowed case A4 for $B_{\{n\}}^{[V]}$ is with $\zeta^{[V]}=0$. It implies the case A3 for $B_{\{n\}}^{[S]}$ with $\kappa^{[S]}=0$. The set G1e in ([68) follows.

- Case A5 for $B_{\{n\}}^{[V]}$ (see Table (1)).

Applying the same reasoning as for the set A1, one finds that the only allowed case for $B_{\{n\}}^{[S]}$ arising from the case A5 for $B_{\{n\}}^{[V]}$ is the case A2 with $\rho_{1}^{[S]}=\rho_{2}^{[S]}=0$. The set G1b in (㟶) follows.

- The results for the Generic Case 1 are summarized in (68) in Sect.(2.5).

\section{A.2 Generic Case 2}

The Generic Case 2, corresponding to $B_{\{n\}}^{[V]} \neq 0$ and $B_{\{n\}}^{[S]}=0$ (158), is compatible with the boundary conditions of all the sets of Table (11) for $[P]=[V]$. 


\section{A.3 Special Cases 1 and 2}

The Special Cases 1 and 2, corresponding to the function $B_{\{n\}}^{[S]}=1$ (59) and respectively to the function $B_{\{n\}}^{[V]} \neq 0$ or $B_{\{n\}}^{[V]}=0$, are compatible with any allowed boundary conditions for $B_{\{n\}}^{[V]}$ and with two subsets only of boundary conditions for $B_{\{n\}}^{[S]}$ (originating from the Sets A1 and A2 of Table (1))

$$
\begin{aligned}
& \text { Boundary Conditions Sa }\left\{\begin{aligned}
B_{\{n\}}^{[S]}(2 \pi R) & =B_{\{n\}}^{[S]}(0)+\alpha_{2}^{[S]} \partial_{s} B_{\{n\}}^{[S]}(0) \\
\partial_{s} B_{\{n\}}^{[S]}(2 \pi R) & =\partial_{s} B_{\{n\}}^{[S]}(0)
\end{aligned}\right. \\
& \text { Boundary Conditions Sb }\left\{\begin{aligned}
\partial_{s} B_{\{n\}}^{[S]}(0) & =0 \\
\partial_{s} B_{\{n\}}^{[S]}(2 \pi R) & =0
\end{aligned}\right.
\end{aligned}
$$


Table 1: Table of allowed boundary conditions for $B_{\{n\}}^{[P]}$ with $[P]=[S]$ or $[P]=[V]$, for all $\{n\}$, from (43))or (44).

\begin{tabular}{|c|l|}
\hline Set & \multicolumn{1}{|c|}{ Boundary Conditions for $B_{\{n\}}^{[P]}$} \\
\hline \hline A1 & $B_{\{n\}}^{[P]}(2 \pi R)=\alpha_{1}^{[P]} B_{\{n\}}^{[P]}(0)+\alpha_{2}^{[P]} \partial_{s} B_{\{n\}}^{[P}(0)$ \\
& $\partial_{s} B_{\{n\}}^{[P]}(2 \pi R)=\alpha_{3}^{[P]} B_{\{n\}}^{[P]}(0)+\alpha_{4}^{[P]} \partial_{s} B_{\{n\}}^{[P]}(0)$ \\
& \multicolumn{1}{|c|}{$\alpha_{1}^{[P]} \alpha_{4}^{[P]}-\alpha_{2}^{[P]} \alpha_{3}^{[P]}=1$} \\
\hline A2 & $\partial_{s} B_{\{n\}}^{[P]}(0)=\rho_{1}^{[P]} B_{\{n\}}^{[P}(0)$ \\
& $\partial_{s} B_{\{n\}}^{[P]}(2 \pi R)=\rho_{2}^{[P]} B_{\{n\}}^{[P]}(2 \pi R)$ \\
\hline A3 & $B_{\{n\}}^{[P]}(0)=0$ \\
& $\partial_{s} B_{\{n\}}^{[P]}(2 \pi R)=\kappa^{[P]} B_{\{n\}}^{[P]}(2 \pi R)$ \\
\hline A4 & $B_{\{n\}}^{[P]}(2 \pi R)=0$ \\
& $\partial_{s} B_{\{n\}}^{[P]}(0)=\zeta^{[P]} B_{\{n\}}^{[P]}(0)$ \\
\hline A5 & $B_{\{n\}}^{[P]}(0)=0$ \\
& $B_{\{n\}}^{[P]}(2 \pi R)=0$ \\
\hline
\end{tabular}


Table 2: Table of Kaluza-Klein vector towers $v_{\{n\}}^{2}>0$ for the Generic Case 2 $\left(B_{\{n\}}^{[V]}(s)=\sigma_{\{n\}}^{[V]} \sin \left(v_{\{n\}} s\right)+\tau_{\{n\}}^{[V]} \cos \left(v_{\{n\}} s\right), B_{\{n\}}^{[S]}(s)=0\right)$. The upper index $[V]$ has been omitted for the parameters $\sigma_{\{n\}}^{[V]}, \tau_{\{n\}}^{[V]}, \alpha_{1}^{[V]}, \ldots$.

\begin{tabular}{|c|c|c|}
\hline \multicolumn{3}{|c|}{$\begin{array}{l}\text { Generic Case } 2\left(B_{\{n\}}^{[S]}=0 \text {, see [58]) }\right. \\
\text { itions and Towers for Real Vector Fields }\end{array}$} \\
\hline Case & Boundary condition & Tower equations \\
\hline gc2-A1-tw & $\begin{array}{l}B_{\{n\}}^{[V]}(2 \pi R)=\alpha_{1} B_{\{n\}}^{[V]}(0)+\alpha_{2} \partial_{s} B_{\{n\}}^{[V]}(0) \\
\partial_{s} B_{\{n\}}^{[V]}(2 \pi R)=\alpha_{3} B_{\{n\}}^{[V]}(0)+\alpha_{4} \partial_{s} B_{\{n\}}^{[V]}(0) \\
\quad \alpha_{1} \alpha_{4}-\alpha_{3} \alpha_{2}=1 \\
\end{array}$ & $\begin{array}{c}v_{\{n\}}\left(\alpha_{1}+\alpha_{4}\right) \cos \left(2 \pi v_{\{n\}} R\right)+\left(\alpha_{2} v_{\{n\}}^{2}-\alpha_{3}\right) \sin \left(2 \pi v_{\{n\}} R\right)-2 v_{\{n\}}=0 \\
\left(\sin \left(2 \pi v_{\{n\}} R\right)-\alpha_{2} v_{\{n\}}\right) \sigma_{\{n\}}=\left(\alpha_{1}-\cos \left(2 \pi v_{\{n\}} R\right)\right) \tau_{\{n\}}\end{array}$ \\
\hline gc2-A2-tw & $\begin{array}{l}\partial_{s} B_{\{n\}}^{[V]}(0)=\rho_{1} B_{\{n\}}^{[V]}(0) \\
\partial_{s} B_{\{n\}}^{[V]}(2 \pi R)=\rho_{2} B_{\{n\}}^{[V]}(2 \pi R)\end{array}$ & $\begin{array}{l}v_{\{n\}}\left(\rho 2-\rho_{1}\right) \cos \left(2 \pi v_{\{n\}} R\right)+\left(v_{\{n\}}^{2}+\rho_{1} \rho_{2}\right) \sin \left(2 \pi v_{\{n\}} R\right)=0 \\
v_{\{n\}} \sigma_{\{n\}}=\rho_{1} \tau_{\{n\}}\end{array}$ \\
\hline gc2-A3-tw & $\begin{array}{l}B_{\{n\}}^{[V]}(0) \quad=0 \\
\partial_{s} B_{\{n\}}^{[V]}(2 \pi R)=\kappa B_{\{n\}}^{[V]}(2 \pi R)\end{array}$ & $\begin{array}{l}v_{\{n\}} \cos \left(2 \pi v_{\{n\}} R\right)-\kappa \sin \left(2 \pi v_{\{n\}} R\right)=0 \\
\quad \tau_{\{n\}}=0\end{array}$ \\
\hline gc2-A4-tw & $\begin{array}{l}B_{\{n\}}^{[V]}(2 \pi R)=0 \\
\partial_{s} B_{\{n\}}^{[V]}(0)=\zeta B_{\{n\}}^{[V]}(0)\end{array}$ & $\begin{array}{c}v_{\{n\}} \cos \left(2 \pi v_{\{n\}} R\right)+\zeta \sin \left(2 \pi v_{\{n\}} R\right)=0 \\
v_{\{n\}} \sigma_{\{n\}}=\zeta \tau_{\{n\}}\end{array}$ \\
\hline gc2-A5-tw & $\begin{array}{l}B_{\{n\}}^{[V]}(0)=0 \\
B_{\{n\}}^{[V]}(2 \pi R)=0\end{array}$ & $\begin{array}{c}\sin \left(2 \pi v_{\{n\}} R\right)=0 \\
\tau_{\{n\}}=0\end{array}$ \\
\hline
\end{tabular}

Table 3: Table of Kaluza-Klein vector states $v_{\{n\}}^{2}=0$ for the Generic Case 2 $\left(B_{\{n\}}^{[V]}(s)=A_{\{n\}}^{[V]} s+B_{\{n\}}^{[V]}, B_{\{n\}}^{[S]}(s)=0\right)$. The upper index $[V]$ has been omitted for the parameters $A_{\{n\}}^{[V]}, B_{\{n\}}^{[V]}, \alpha_{1}^{[V]}, \ldots$.

\begin{tabular}{|c|c|c|}
\hline \multicolumn{3}{|c|}{$\begin{array}{c}\text { Generic Case } 2\left(B_{\{n\}}^{[S]}=0 \text {, see }(\overline{58})\right. \\
\text { oundary Conditions for a Real Vector State with } v_{\{n\}}=0\end{array}$} \\
\hline Case & "Boundary condition & Tower equations \\
\hline gc2-A1-0 & $\begin{array}{c}B_{\{n\}}^{[V]}(2 \pi R)=\alpha_{1} B_{\{n\}}^{[V]}(0)+\alpha_{2} \partial_{s} B_{\{n\}}^{[V]}(0) \\
\partial_{s} B_{\{n\}}^{[V]}(2 \pi R)=\alpha_{3} B_{\{n\}}^{[V]}(0)+\alpha_{4} \partial_{s} B_{\{n\}}^{[V]}(0) \\
\alpha_{1} \alpha_{4}-\alpha_{3} \alpha_{2}=1 \\
\end{array}$ & $\begin{array}{c}\left(\alpha_{1}+\alpha_{4}-2\right)-2 \pi R \alpha_{3}=0 \\
\left(1-\alpha_{4}\right) A=\alpha_{3} B\end{array}$ \\
\hline gc2-A2-0 & $\begin{array}{l}\partial_{s} B_{\{n\}}^{[V]}(0)=\rho_{1} B_{\{n\}}^{[V]}(0) \\
\partial_{s} B_{\{n\}}^{[V]}(2 \pi R)=\rho_{2} B_{\{n\}}^{[V\}}(2 \pi R)\end{array}$ & $\begin{array}{c}\left(\rho 1-\rho_{2}\right)-2 \pi R \rho_{1} \rho_{2}=0 \\
A=\rho_{1} B\end{array}$ \\
\hline gc2-A3-0 & $\begin{aligned} B_{\{n\}}^{[V]}(0) & =0 \\
\partial_{s} B_{\{n\}}^{[V]}(2 \pi R) & =\kappa B_{\{n\}}^{[V]}(2 \pi R)\end{aligned}$ & $\begin{array}{c}2 \pi R \kappa-1=0 \\
B=0\end{array}$ \\
\hline gc2-A4-0 & $\begin{array}{l}B_{\{n\}}^{[V]}(2 \pi R)=0 \\
\partial_{s} B_{\{n\}}^{[V]}(0)=\zeta B_{\{n\}}^{[V]}(0)\end{array}$ & $\begin{array}{c}2 \pi R \zeta+1=0 \\
A=\zeta B\end{array}$ \\
\hline gc2-A5-0 & $\begin{array}{l}B_{\{n\}}^{[V]}(0)=0 \\
B_{\{n\}}^{[V]}(2 \pi R)=0\end{array}$ & impossible \\
\hline
\end{tabular}


Table 4: Table of Kaluza-Klein vector state $v_{\{n\}}^{2}<0\left(w_{\{n\}}^{2}=-v_{\{n\}}^{2}>\right.$ $0)$ for the Generic Case $2\left(B_{\{n\}}^{[V]}(s)=\sigma_{\{n\}}^{[V]} \sinh \left(w_{\{n\}} s\right)+\tau_{\{n\}}^{[V]} \cosh \left(w_{\{n\}} s\right)\right.$, $\left.B_{\{n\}}^{[S]}(s)=0\right)$. The upper index $[V]$ has been omitted for the parameters $\sigma_{\{n\}}^{[V]}, \tau_{\{n\}}^{[V]}, \alpha_{1}^{[V]}, \ldots$.

\begin{tabular}{|c|c|c|}
\hline \multicolumn{3}{|c|}{$\begin{array}{c}\text { Generic Case } 2\left(B_{\{n\}}^{[S]}=0, \text { see } 58\right) \\
\text { oundary Conditions and Towers for a Real Vector State with } w_{\{n\}}^{2}=-v_{\{n\}}^{2}>0\end{array}$} \\
\hline 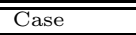 & "Boundary condition & Tower equations \\
\hline gc2-A1-tac & $\begin{array}{c}B_{\{n\}}^{[V]}(2 \pi R)=\alpha_{1} B_{\{n\}}^{[V]}(0)+\alpha_{2} \partial_{s} B_{\{n\}}^{[V]}(0) \\
\partial_{s} B_{\{n\}}^{[V]}(2 \pi R)=\alpha_{3} B_{\{n\}}^{[V\}}(0)+\alpha_{4} \partial_{s} B_{\{n\}}^{[V\}}(0) \\
\alpha_{1} \alpha_{4}-\alpha_{3} \alpha_{2}=1\end{array}$ & $\begin{array}{c}w_{\{n\}}\left(\alpha_{1}+\alpha_{4}\right) \cosh \left(2 \pi w_{\{n\}} R\right)-\left(\alpha_{2} w_{\{n\}}^{2}+\alpha_{3}\right) \sinh \left(2 \pi w_{\{n\}} R\right)-2 w_{\{n\}}=0 \\
\left(\sinh \left(2 \pi w_{\{n\}} R\right)-\alpha_{2} w_{\{n\}}\right) \sigma_{\{n\}}=\left(\alpha_{1}-\cosh \left(2 \pi w_{\{n\}} R\right)\right) \tau_{\{n\}}\end{array}$ \\
\hline gc2-A2-tac & $\begin{array}{l}\partial_{s} B_{\{n\}}^{[V]}(0)=\rho_{1} B_{\{n\}}^{[V]}(0) \\
\partial_{s} B_{\{n\}}^{[V]}(2 \pi R)=\rho_{2} B_{\{n\}}^{[V\}}(2 \pi R)\end{array}$ & $\begin{array}{l}w_{\{n\}}\left(\rho_{1}-\rho_{2}\right) \cosh \left(2 \pi w_{\{n\}} R\right)+\left(w_{\{n\}}^{2}-\rho_{1} \rho_{2}\right) \sinh \left(2 \pi w_{\{n\}} R\right)=0 \\
w_{\{n\}} \sigma_{\{n\}}=\rho_{1} \tau_{\{n\}}\end{array}$ \\
\hline gc2-A3-tac & $\begin{array}{l}B_{\{n\}}^{[V]}(0)=0 \\
\partial_{s} B_{\{n\}}^{[V]}(2 \pi R)=\kappa B_{\{n\}}^{[V]}(2 \pi R)\end{array}$ & $\begin{array}{l}w_{\{n\}} \cosh \left(2 \pi w_{\{n\}} R\right)-\kappa \sinh \left(2 \pi w_{\{n\}} R\right)=0 \\
\tau_{\{n\}}=0\end{array}$ \\
\hline gc2-A4-tac & $\begin{array}{l}B_{\{n\}}^{[V]}(2 \pi R)=0 \\
\partial_{s} B_{\{n\}}^{[V]}(0)=\zeta B_{\{n\}}^{[V]}(0)\end{array}$ & $\begin{array}{c}w_{\{n\}} \cosh \left(2 \pi w_{\{n\}} R\right)+\zeta \sinh \left(2 \pi w_{\{n\}} R\right)=0 \\
w_{\{n\}} \sigma_{\{n\}}=\zeta \tau_{\{n\}}\end{array}$ \\
\hline gc2-A5-tac & $\begin{array}{l}B_{\{n\}}^{[V]}(0)=0 \\
B_{\{n\}}^{[V]}(2 \pi R)=0\end{array}$ & $\begin{array}{c}\sinh \left(2 \pi w_{\{n\}} R\right)=0 \rightarrow \text { impossible } \\
\tau_{\{n\}}=0\end{array}$ \\
\hline
\end{tabular}

